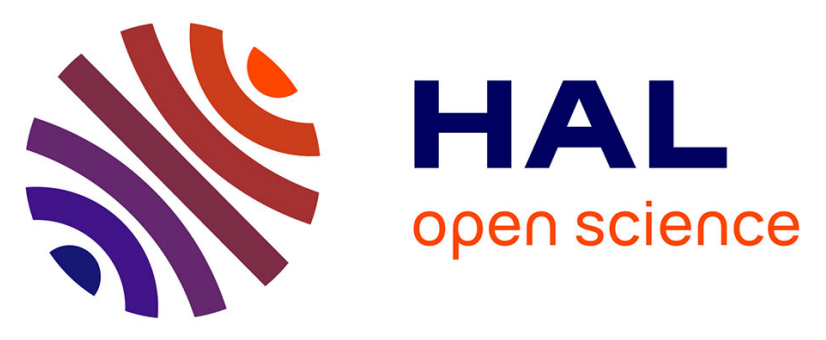

\title{
Expert system dedicated to condition-based maintenance based on a knowledge graph approach: Application to an aeronautic system
}

Alexandre Sarazin, Jérémy Bascans, Jean-Baptiste Sciau, Jiefu Song, Bruno Supiot, Aurelie Montarnal, Xavier Lorca, Sébastien Truptil

\section{To cite this version:}

Alexandre Sarazin, Jérémy Bascans, Jean-Baptiste Sciau, Jiefu Song, Bruno Supiot, et al.. Expert system dedicated to condition-based maintenance based on a knowledge graph approach: Application to an aeronautic system. Expert Systems with Applications, 2021, 186, pp.1-10/115767. 10.1016/j.eswa.2021.115767 . hal-03333514

\section{HAL Id: hal-03333514 https://imt-mines-albi.hal.science/hal-03333514}

Submitted on 20 Sep 2021

HAL is a multi-disciplinary open access archive for the deposit and dissemination of scientific research documents, whether they are published or not. The documents may come from teaching and research institutions in France or abroad, or from public or private research centers.
L'archive ouverte pluridisciplinaire HAL, est destinée au dépôt et à la diffusion de documents scientifiques de niveau recherche, publiés ou non, émanant des établissements d'enseignement et de recherche français ou étrangers, des laboratoires publics ou privés. 


\title{
Expert system dedicated to condition-based maintenance based on a knowledge graph approach: Application to an aeronautic system
}

\author{
Alexandre Sarazin ${ }^{\mathrm{a}, \mathrm{b}, *}$, Jérémy Bascans ${ }^{\mathrm{a}}$, Jean-Baptiste Sciau ${ }^{\mathrm{c}}$, Jiefu Song ${ }^{\mathrm{c}}$, Bruno Supiot ${ }^{\mathrm{c}}$, \\ Aurélie Montarnal ${ }^{\mathrm{b}}$, Xavier Lorca ${ }^{\mathrm{b}}$, Sébastien Truptil ${ }^{\mathrm{d}}$ \\ a Data Processing \& AI Division, APSYS, Blagnac, France \\ ${ }^{\mathrm{b}}$ Centre Génie Industriel, IMT Mines Albi, Albi, France \\ ${ }^{\mathrm{c}}$ Recherche et Développement, ACTIVUS, Toulouse, France \\ d CEA, CEA Tech Occitanie, Labège, France
}

Keywords:

CBM

Expert System

Knowledge graph

\begin{abstract}
A B S T R A C T
Condition Based Maintenance (CBM) has become the focus of many research topics over the past decades. This is mostly related to the development of new machine learning algorithms and the ever increasing capacity to collect data allowing failures to be detected and the system's remaining lifetime to be estimated while requiring few or no expert knowledge. However, current machine learning based CBM solutions have limitations. They require an extensive and relevant data set to train on and are performed at the component level, not systemwide. Conversely, Expert Systems (ES) do not have these restrictions but should be used on systems with available expert knowledge and are currently suffering from efficiency, scalability and applicability limits. In this paper, an ES solution for CBM based on an heterogeneous information network will be presented to address the efficiency, scalability and applicability issues of modern ES. An application to an aircraft system will be used as case study to illustrate the process and performance of this solution for anomaly detection and diagnostics.
\end{abstract}

\section{Motivation}

The increasing complexity of modern manufacturing systems emphasizes the need for preventive maintenance. At the same time, reducing maintenance costs has become a critical challenge in many industries like transportation (Numanoğlu \& Ekmekçi, 2020) or energy (Salameh et al., 2018). Considering this policy, traditional timebased maintenance can be lacking, creating a need for more cost effective condition-based maintenance (CBM) solutions (Bayoumi et al., 2008; Gündüz et al., 2019; Huynh, 2020).

CBM is based on sensor data analysis in order to detect symptoms and failures, sometimes estimating the systems remaining useful life (RUL). Two main approaches are being used to process sensor data. A first approach is to feed learning algorithms with labeled historical data to solve classification or regression problems from past experience. The main advantage of this solution is its high adaptability as no need for system knowledge is required. Even though a lot of works and applications have been made on supervised machine learning algorithms, the availability and high quality requirements on the training dataset are inherent major issues (Sheng \& Zhang, 2019). The solution proposed in this paper focuses on CBM for systems with few available training sets and lots of human expertise or available documentation. Therefore, the machine learning approach has been discarded here. A second approach to diagnose failures is to capitalize on existing system knowledge to artificially re-create an expert reasoning to reach a conclusion by applying predefined rules related to monitored facts. These systems are being referred to as Expert Systems (ES).

The purpose of this paper is to propose an architecture for CBM addressing current ES limitations. After presenting the related state of the art and its current limitations, a solution will be detailed, experimented and finally discussed.

\section{Literature review}

As defined by ISO (AFNOR, 2018), maintenance is a "set of activities or tasks used to restore an item to a state in which it can perform its designated functions". It can be divided into two main strategies:

\footnotetext{
* Corresponding author at: Data Processing \& AI Division, APSYS, Blagnac, France.

E-mail addresses: alexandre.sarazin@apsys-airbus.com (A. Sarazin), jeremy.bascans@apsys-airbus.com (J. Bascans), jeanbaptiste.sciau@activus-group.fr (J.-B. Sciau), jiefu.song@activus-group.fr (J. Song), bruno.supiot@activus-group.fr (B. Supiot), aurelie.montarnal@mines-albi.fr (A. Montarnal), xavier.lorca@mines-albi.fr (X. Lorca), sebastien.truptil@cea.fr (S. Truptil).
} 
corrective and preventive. While corrective maintenance consists in restoring (repairing or replacing) some equipment to its required function after it has failed, preventive maintenance evaluates and/or slows the equipment's degradation, reducing its probability of failure. During the last ten years, due to the increase of sensor data collection, lots of Research focused on a specific type of preventive maintenance named CBM, which evaluates and analyzes the equipment's physical conditions while considering the potential maintenance actions to perform.

In particular, Prognostics and Health Management (PHM) is a kind of CBM introduced as a "discipline consisting of technologies and methods to assess the reliability of a product in its actual life cycle conditions to determine the advent of failure and mitigate equipment/system risk" (Cheng et al., 2010). This approach consists of three main steps: the monitoring of the system, usually using sensors, the failure identification, consisting in a diagnostics step, and the prognostics step which evaluates the system's remaining useful life (RUL) (Jouin et al., 2013).

Several PHM-like solutions have been designed for aircrafts and gas turbine engines in particular. Roemer and Kacprzynski (2000) proposes a review of diagnostics and prognostics solutions with an emphasis on data processing techniques ensuring the input records reliability. Hanachi et al. (2018) presents another example of health monitoring, diagnostics and prognostics approach based on performance data. However, while these works detail numerous relevant techniques for data collection, failure diagnostics and prognostics, none of them propose an architecture integrating these algorithms. In particular, expert knowledge management still needs to be addressed.

The failure identification is a critical step to select the corresponding degradation model and provide accurate information on the root cause to the user. As a matter of fact, the process of relating sensor data to failures is already performed in diagnostic systems based on the technical documentation and human expertise. The difference between diagnostics and prognostics is that diagnostics is performed after the advent of failure while prognostics estimates the RUL of the system from signs detected before the failure (Lee et al., 2014).

According to Zaidan et al. (2015) statistically robust methodologies are more appropriate to deal with large amounts of data in real time for complex systems due to the difficulty in designing physical degradation models. Consequently a Bayesian approach is proposed for prognostics and improved in Zaidan et al. (2016). The Monte-Carlo approach can also be considered as a probabilistic and stochastic solution (Puggina \& Venturini, 2012).

Jackson (1998) introduces an expert system as a software integrating expert knowledge on a specific domain in order to solve problems and provide advice by reproducing an expert reasoning based on facts and data. According to this definition, the solution proposed in this paper belongs to the expert system's field. As such, it should be composed of three elements: a base of facts, a base of rules and a control structure (Levine \& Pomerol, 1990). The base of facts is a set of atomic elements of knowledge available before the reasoning. The base of rules regroups all the rules to apply to the base of facts in order to reach a conclusion. These rules can be provided by a human expert or a technical documentation and performed if the status of the base of facts satisfies its activation conditions. The control structure is designed to choose which rule should be activated should a conflict arise due to several rules activation conditions being fulfilled.

As expert systems have been defined several decades ago, many applications have been designed especially in the medical field. Indeed, the process of a physician identifying a disease from symptoms on a patient is similar to the process of identifying faults from anomalies in a system. Consequently the maintenance and medicine fields contributed greatly to ES development. Yanase and Triantaphyllou (2019) proposes a survey on computer-aided diagnosis in medicine with an entire section describing ES structure and algorithms with related works. AbuNasser (2017) details a different description of ES components and lists 33 medical works based on ES. Rajabi et al. (2019) presents a statistical study of ES works in the medical field over 30 years. It concludes that
"AI and expert systems have a high potential to be employed in almost all fields of medicine".

The critical criteria to evaluate an ES performance is knowledge management. However, according to Xiaoxue et al. (2019), current knowledge management techniques have some "common defects in efficiency, scalability and applicability". For instance, modern conditionbased systems are supported by technical data increasingly complex, dynamic, semantic and often updated. Knowledge graphs can address these limitations. A knowledge graph can be defined as a "structured semantic knowledge base that describes concepts and their relationships in the physical world in the form of symbols. It allows knowledge representation and management to solve knowledge association problems, such as knowledge retrieval and semantic question answering. It is the basis and bridge to realize intelligent semantic retrieval" (Xiaoxue et al., 2019). Knowledge graphs can be implemented using different solutions like Bayesian networks, conceptual graphs or heterogeneous information networks (HIN).

According to Agrawal et al. (2000), Naive Bayes classifiers are recognized to be among the best for classifying text. As such it can be used to create, process, and maintain a hierarchical arrangement of textual documents through interactive mining-based operations. Bayesian networks can also be used in maintenance diagnostic and decisionmaking systems although models should be implemented to grant an improved performance over time (Corset et al., 2003).

Conceptual graph are knowledge notations processed by reasoning operators defined by Sowa (1983). It was designed to address problems related to natural language processing, database inference, and knowledge engineering. This work was further improved by numerous researches. Roussey (2001) implemented the notions of vocabulary and label while Chein et al. (1998) introduced the concept of nested graphs.

HIN are defined by Sun and Han (2012) as an abstraction of the real world, focusing on the objects and the interactions between the objects. The network structure of HIN provides an increased scalability when increasing the system's complexity as the knowledge processing and intelligibility is improved. Davis et al. (2011) presents three different implementations of HIN with a focus on supervised and unsupervised link prediction in a multi-relational setting. Other examples can be found in Angelova et al. (2012) to address the problem of multi-label classification in heterogeneous graphs, where nodes belong to different types and different types have different sets of classification labels.

In order to structure maintenance data in a CBM information network, a metamodel is required. This metamodel should define the functional nature of the different kinds of nodes and edges. In this paper, the work of Guillén et al. (2016) has been used as a basis. In this metamodel, the maintenance data is structured in 5 blocks:

- A physical description block describes the systems components from a perspective similar to a bill of materials. The smallest components maintained are called Maintainable Items (MIs).

- A functional description block defines the functions performed by a component or subsystem and the corresponding failures they are subjected to.

- An information source block gathers information on the system's monitoring from heterogeneous sources like sensors or a maintenance information system. These data are then being transformed into monitoring variables using measurement techniques.

- A symptom analysis block designed to detect anomalies in the monitoring variables and relate it to a symptom through descriptors. Depending on the descriptors output, interpretation rules can trigger 3 different kinds of actions: detection, diagnosis or prognosis.

- A maintenance decision-making block composed of a set of detection, diagnosis and prognostics processes applied based on the other four blocks. These processes outputs can be aggregated and sent to a maintenance decision process to provide valuable information to the end user. 
As a conclusion, this literature review defined the CBM and PHM concepts and detailed the components of most ES. From the metamodel presented, a CBM-oriented ES can be designed. However as most modern ES have limitations in efficiency, scalability and applicability, a solution should be proposed to address these deficiencies. In this perspective, a framework integrating a knowledge graph to support the ES base of facts is proposed.

\section{Contribution}

As previously mentioned, most of recent works on CBM focus on data collection, failure diagnostics and prognostics techniques but research on architectures able to integrate these algorithms is lacking. Expert knowledge management is especially challenging. The main limitations to modern ES is the complexity of managing knowledge which induces efficiency, scalability and applicability problems (Xiaoxue et al., 2019).

In this paper, a solution to implement CBM will be proposed. This solution should be designed to integrate human expertise and technical data to perform diagnostics on complex systems, with few occurrences of each failure. Furthermore, it should integrate heterogeneous data from different sources: technical documentation, monitoring data and historical maintenance records. Consequently, the choice of an ES based solution has been made with the perspective of extending it to a PHM solution. In order to address the efficiency, scalability and applicability issues, this paper proposes an ES solution based on HIN to represent and exploit heterogeneous data (sensor data, maintenance records, technical data) from different sources (sensors, maintenance information system, technical documentation). The choice of HIN has been motivated by the criteria illustrated in Table 1 .

In this section, we have argued the selection of a HIN expert system for CBM and structured its knowledge base concepts with a CBM-specific meta-model.

Section 4 will detail the solution used to implement the expert system (ES) base of facts, base of rules and control structure in a HIN. In part 5 , Section 5 will introduce a real case study on an aircraft system. For this case study, a focus has been made on diagnostic in order to identify a defective component from a detected failure. Section 6 of this paper will propose to implement a probabilistic model on the network in order to improve the diagnostic performance. Finally, the results will be presented and discussed within Section 7 .

\section{Knowledge graph based expert system}

In the previous sections, the concepts behind CBM, ES and knowledge graphs have been presented and the motivation to design a HIN based ES has been explicited as a solution to address the efficiency, scalability and applicability limitations of current ES. To describe this solution, the base of facts will first be presented as a HIN. Next, the base of rules implementation and their application process through the control structure will be detailed.

\subsection{Base of facts designed from HIN}

According to Levine and Pomerol (1990), an ES base of facts is a set of elementary chunks of knowledge available before the reasoning. It can also be referred to as the ES knowledge base (Liebowitz, 1995). In this paper, the structure chosen to implement the base of facts is the HIN. Sun and Han (2012) defines a information network as "a directed graph $G=(V, E)$ with an object type mapping function $\tau: V \rightarrow A$ and a link type mapping function $\phi: E \rightarrow R$, where each object $v \in V$ belongs to one particular object type $\tau(v) \in A$, each link $e \in E$ belongs to a particular relation $\phi(e) \in R$, and if two links belong to the same relation type, the two links share the same starting object type as well as the ending object type". If there is more than a single type of object or relation, the information network is heterogeneous.

The variables involved in the proposed meta-model are as follows:
- Sets are uppercase latin letters.

- Values are lowercase greek symbols.

- Functions are lowercase latin letters.

Finally, G is a graph with: $G=(V, E)$, where $V=\left\{v_{1}, \ldots, v_{u}\right\}$ is the set of nodes, and $E=\left\{\epsilon_{1}, \ldots, \epsilon_{e}\right\}$ the set of edges with

$E=\left\{\epsilon_{k}=\left(v_{i}, v_{j}\right) \in V^{2},(i, j) \in[1 ; u]^{2}, i \neq j\right\}$

- Each node has a type $\tau$ which belongs to $T=\left\{\tau_{1}, \ldots, \tau_{t}\right\}$. The function $f_{v}: v \rightarrow \tau$ is used to return the type $\tau$ of node $v$.

- Each edge has a type $\mu$ belonging to the set $M=\left\{\mu_{1}, \ldots, \mu_{m}\right\}$. The function $f_{\epsilon}: \epsilon \rightarrow \mu$ returns the type $\mu$ of an edge $\epsilon$.

This formalization can be used to define the nodes and edges characteristics with their types. These types can indeed be specified using a set of attributes from the whole $X=\left\{\chi_{1}, \ldots, \chi_{x}\right\}$. These attributes are properties and/or characteristics representing the nodes and edges types. These attributes can be customized for any type of node or edge depending on the information requirements to implement and exploit.

All attributes of a node type $\tau$ can be returned by the function

$f_{\tau}: \tau \rightarrow X_{j}$ with $X_{j} \subset X$.

Likewise, all attributes of an edge type $\mu$ can be returned by

$f_{\mu}: \mu \rightarrow X_{j}$ with $X_{j} \subset X$.

For two nodes $v_{p}$ and $v_{n}$ and an edge type $\mu_{l}$, the function $g$ (4) returns a concrete edge $\epsilon_{x}$ if it exists and (5) the set $\left\{\epsilon_{1}, \ldots, \epsilon_{a}\right\}$ representing all real edges between two nodes $v_{p}$ and $v_{n}$ with $\left\{\epsilon_{1}, \ldots, \epsilon_{a}\right\} \subset E$ without constraint on edge type.

$g: v_{p} * v_{n} * \mu_{l} \rightarrow \epsilon_{x}$

$g: v_{p} * v_{n} \rightarrow\left\{\epsilon_{1}, \ldots, \epsilon_{a}\right\}$

The value $\alpha$ of an attribute $\chi$ for a node $v$ is returned by the function:

$h_{v}: v * \chi \rightarrow \alpha$

Likewise, the value $\beta$ of an attribute $\chi$ of the edge $\epsilon$ is returned by the function:

$h_{\epsilon}: \epsilon * \chi \rightarrow \beta$

Finally, these values can be modified using the function $l$ :

$l_{v}: \chi * v * \alpha \rightarrow v$ for a node $v$

$l_{\epsilon}: \chi * \epsilon * \alpha \rightarrow \epsilon$ for an edge $\epsilon$

\subsection{Rules integration}

Once the base of facts has been defined, the rules and their application process should be detailed. To build a complex expert system, various rules should be integrated such as $R=\left\{\gamma_{1}, \ldots, \gamma_{n}\right\}$. Each rule $\gamma_{i}$ is represented and integrated using a combination of pre-defined declarative algebraic operator. These operators allow to:

- integrate new knowledge (i.e. add new nodes),

- correlate knowledge (i.e. add new edges)

- navigate through the graph (i.e. search paths in the graph depending on rules)

The rules application process proposed in this paper is illustrated in Fig. 1. With each modification of the ES base of facts, the related nodes and edges are identified. The modifications can either be creation, deletion or updates of nodes and/or edges. Browsing through the graph to identify the related nodes and edges can be performed using a read method. From this sub-graph, a set of rules can be extracted using a projection operator. 
Table 1

Comparison table for KG solutions.

\begin{tabular}{|c|c|c|c|c|c|c|c|}
\hline Knowledge graph solution & Genericity & Heterogeneity & Intelligibility & Adaptability & Upgradability & Volumetry & Velocity \\
\hline Bayesian networks & $\checkmark$ & $x$ & $x$ & $\checkmark$ & $\checkmark$ & $x$ & $x$ \\
\hline Conceptual graphs & $\checkmark$ & $x$ & $\checkmark$ & $\checkmark$ & $\checkmark$ & $x$ & $x$ \\
\hline HIN & $\checkmark$ & $\checkmark$ & $\sqrt{ }$ & $\sqrt{ }$ & $\checkmark$ & $\checkmark$ & $\checkmark$ \\
\hline
\end{tabular}

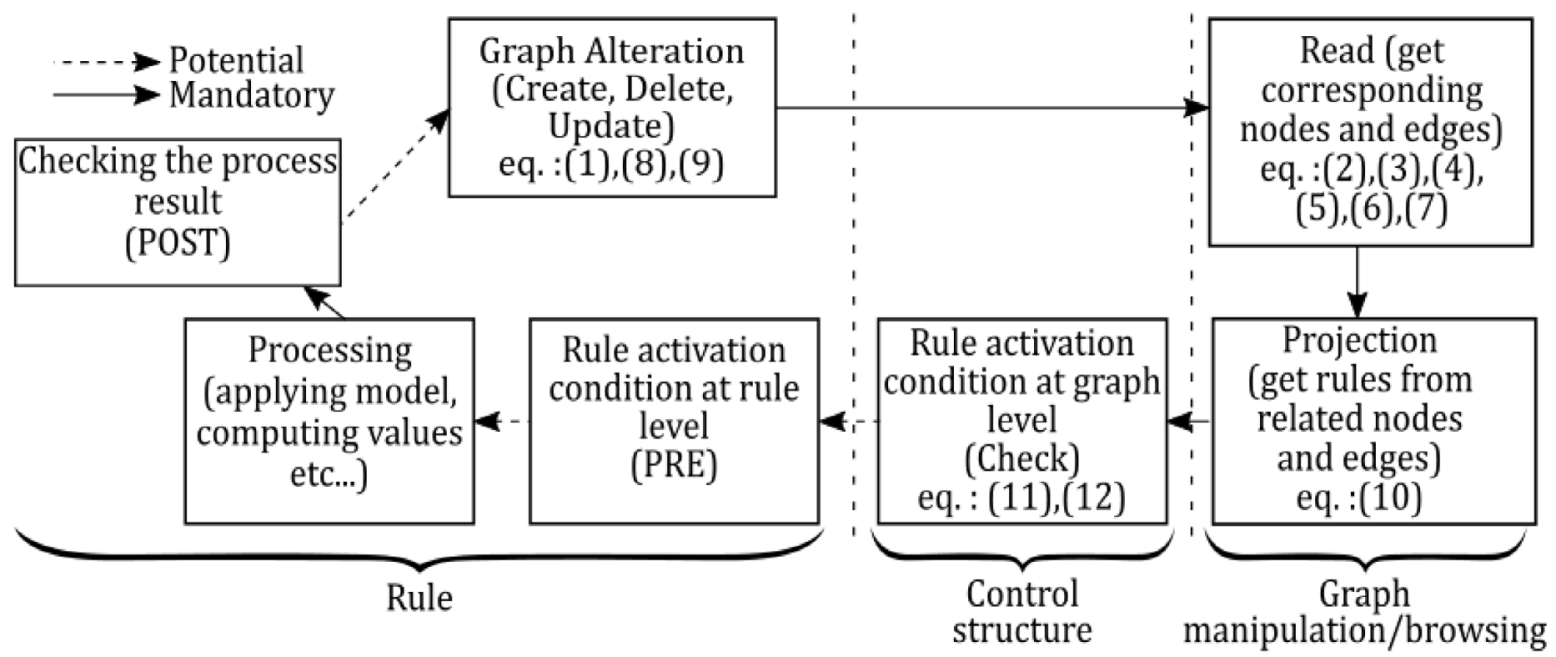

Fig. 1. Rules processing referencing related equations.

The Fig. 2 illustrates an example of graph. The node $v_{1}$, of type $\tau_{1}$, is related to the nodes $v_{3}$ and $v_{4}$, of type $\tau_{2}$, respectively by the edges $\epsilon_{1}$ and $\epsilon_{2}$ of type $\mu_{1}$. The node $v_{2}$, of type $\tau_{1}$, is related to the node $v_{4}$ by the edge $\epsilon_{3}$ of type $\mu_{1}$. Nodes of the same type share the same kinds of attributes noted $\chi_{1}$ and $\chi_{2}$. Each edge can have a rule $\gamma$. To explain this with a real world example, the situation of a systems' overheat can be considered. In this case, the overheat is caused by the failure of one of two fans. The node "Overheat", of type "FailureMode", is related to the nodes "Fan1" and "Fan2", of type "MaintenableItem" (MI), by the edges "P1-F1" and "P1-F2", of type "IsCausedBy". Each node has an attribute "ID" and the MIs also have a "Lifetime". This lifetime impacts the rule "Probability" of the edges which indicates the most probable cause for the failure.

A projection operator provides all rules on the connection between two nodes through an edge type $\mu$. In the example presented in Fig. 2, a project applied to the nodes $v_{1}$ and $v_{2}$ related by the edge $\epsilon_{1}$ would return the rule $\gamma_{1}$. The general formula is expressed as:

Project $_{v v}: v_{a} * v_{b} * \mu \rightarrow R_{j}$ with $R_{j} \subset R$

A modification of the ES base of facts can trigger several rules. Preventing conflicts to arise is the purpose of the control structure. It is performed here using a check operator which applies a constraint type rule to attributes of a set of nodes or edges. The check result is the list of rules to apply. Using the example given in Fig. 2, one such rule could be that only the nodes of type $\tau_{2}$ with the maximum number of related nodes of type $\tau_{1}$ meeting predefined conditions on attribute $\chi_{1}$, like a threshold, have their rules applied on the edges. For instance, if the attributes $\chi_{1}$ of the nodes $v_{1}$ and $v_{2}$ fulfill the conditions, the rules $\gamma_{2}$ and $\gamma_{3}$ are returned by the check function but not $\gamma_{1}$ as $v_{3}$ is only related to a single node $v_{1}$. A general formula is proposed as:

$$
\begin{aligned}
& \text { Check }_{V}: X_{i} * V_{j} * R_{k} \rightarrow R_{k}^{\prime} \text { with } X_{i} \subset X, V_{j} \subset V, R_{k}^{\prime} \subset R_{k} \subset R \\
& \text { Check }_{E}: X_{i} * E_{j} * R_{k} \rightarrow R_{k}^{\prime} \text { with } X_{i} \subset X, E_{j} \subset E, R_{k}^{\prime} \subset R_{k} \subset R
\end{aligned}
$$

A rule is composed of two main parts: the activation condition and the effect. Only when the activation condition is fulfilled can further processing be performed. Some preprocessing may be necessary at this step. A first processing step can then compute values using complex models or algorithms. The result may be checked in a post processing step before altering the graph. This graph alteration can consist in creating or deleting nodes and/or edges. Some attributes values can also be updated. These modifications can then trigger a new read and repeat the process.

Using this process, the rules from the ES base of rules can be applied to the base of facts structured in a HIN while preventing conflicts. Due to the cyclic behavior of the rule's application process, an acyclic graph is required to reduce the risk of getting into infinite loops. This generic structure should now be used to implement a CBM solution with a case study.

\section{Application to aircraft complex systems}

Once the rules' implementation has been theoretically defined, it should be experimented in a real case study. In this paper, the HIN based ES has been applied to an aircraft system. In order to create the HIN, the metamodel presented in Guillén et al. (2016) has been adapted according to Fig. 3. In this adapted metamodel, the maintenance decision block was not used as the diagnosis, prognosis, detection and maintenance decision elements are processes to implement on the HIN which do not store information. The functions and functional failures also disappeared because the relation between failure mode and are considered sufficient.

The detection process is meant to generate symptom and failure warnings based on sensor data analysis. Consequently, the "value" attribute of the "Monitoring Variable" nodes should be updated over time. Following the process detailed in the previous section, the descriptor values' attribute should be computed an updated using the descriptors' formula. Following this update, a second rule may turn the symptoms' activated attribute to true if the condition set from the interpretation rules' rules attribute is met. If all the activated attribute value of all the symptom related to a failure mode are true, then the failure modes' activated attribute is set to true.

The diagnosis process should identify which Maintainable Item (MI) is most probably the cause when a failure occurs. This process has been performed through four steps as described in Fig. 4. Its implementation should be composed of a set of rules applied to the relations between 


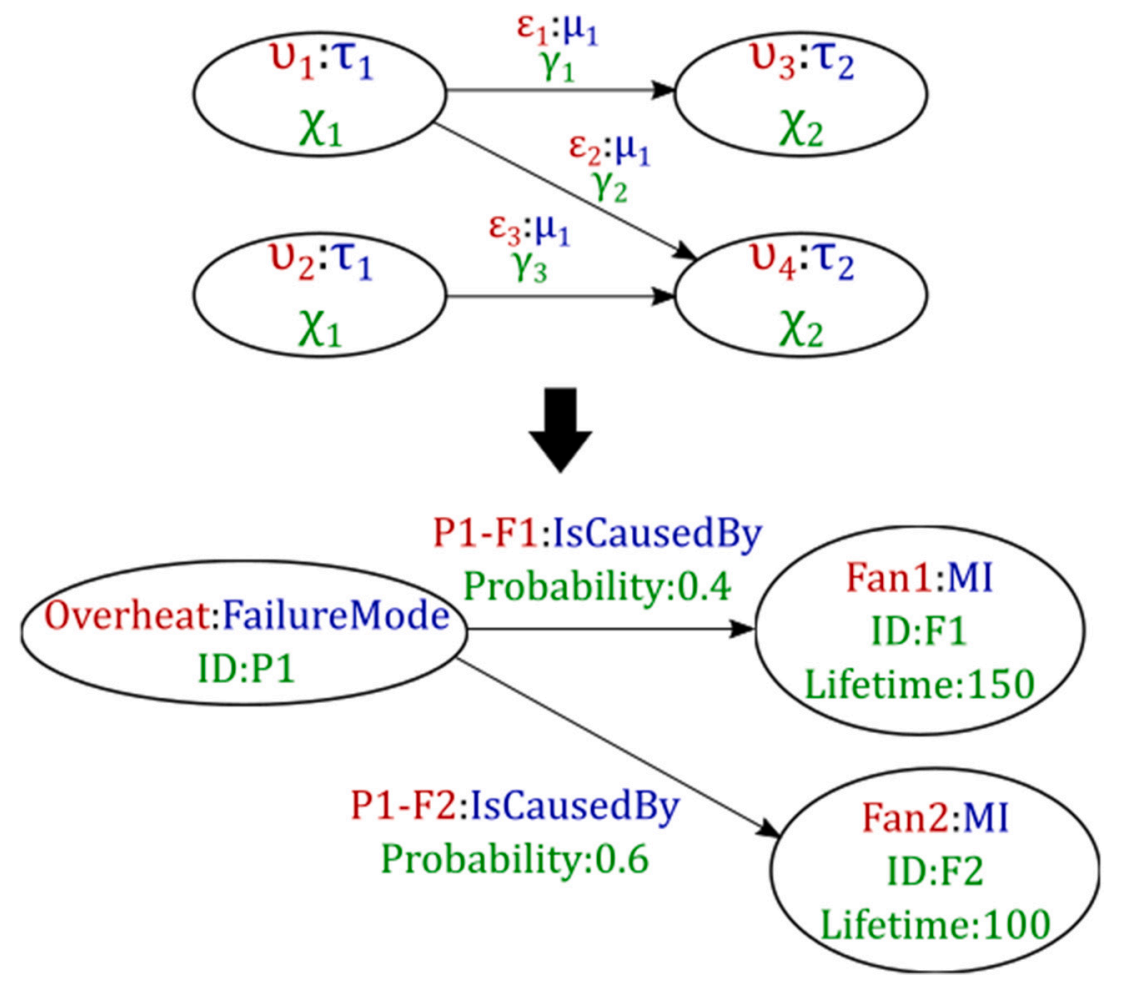

Fig. 2. Graph example.

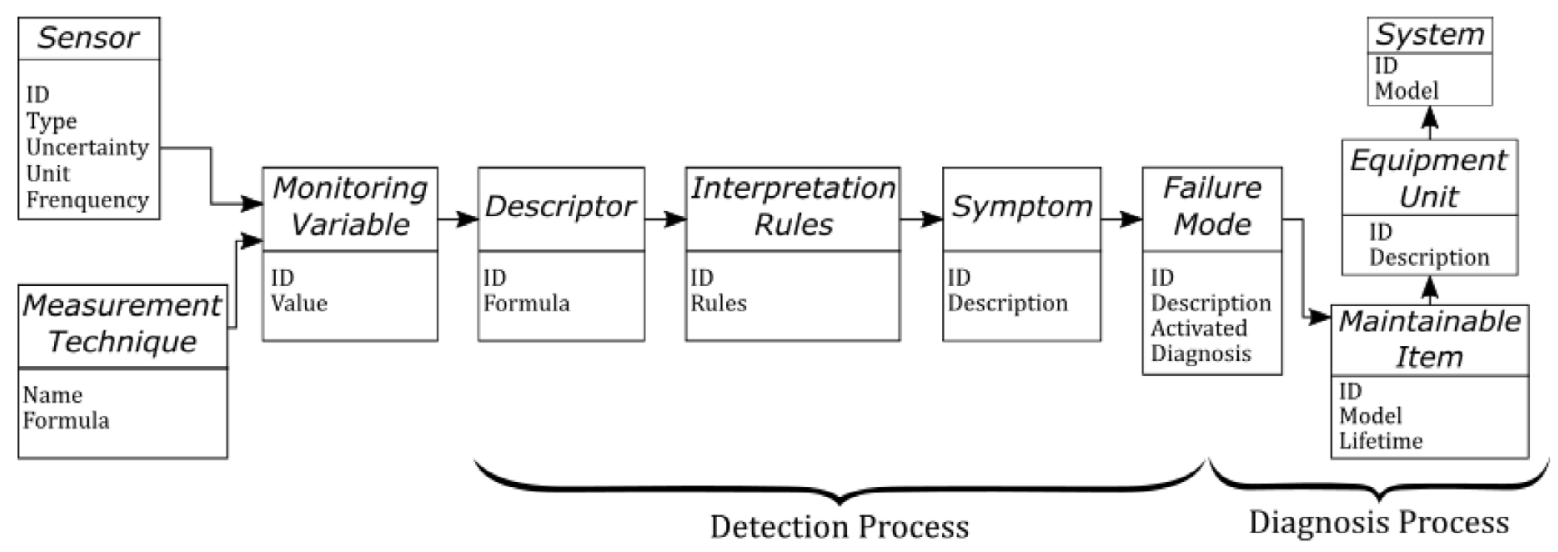

Fig. 3. KG metamodel.

a failure node and the related MI nodes. When a single failure mode is related to several MI, some processing should be performed to sort them depending on their reliability.

In order to create this HIN, documentation data have been implemented following this metamodel. While the considered system is not a whole aircraft but only a subsystem, the resulting graph is composed of more than 4000 nodes and more than 12000 relations. It should be mentioned that only physical MI are considered in this paper as software components cannot be maintained using the same logic. In addition to documentation data, maintenance data can be implemented in the graph. For instance, the MI model can change over time and the MI lifetime increases every time the aircraft flies and is reinitialized every time the equipment is changed or repaired.

In this section, rules have been applied to an aircraft system according to Section 3. A first analysis of this HIN from a diagnostic perspective points that $33 \%$ of the graphs failures are related to a single MI. Consequently, for these failures, simple propagation is enough to identify the cause and no further analysis is required. However, for the other $77 \%$, more processing should be performed in order to identify the defected MI. In this paper, an implementation of probabilistic rules based on Weibull distribution is proposed to sort the MI by probability of failure.

\section{Extension to probabilistic rules}

\subsection{Motivation}

Even though, in this case study, $43 \%$ of failure modes are related to a single MI, in most cases several MI are possibly responsible. In reality, airlines operators often manage to directly identify the failed equipment from the failure modes based on their experience and past occurrences. Following this logic, an expert rule has been implemented in the graph based on a probabilistic approach from an analysis of past failure occurrences. The aim is to sort the MIs related to the Failure Mode by decreasing failure probability. Through this process, the MI with the highest probability of breaking down can be identified. In 


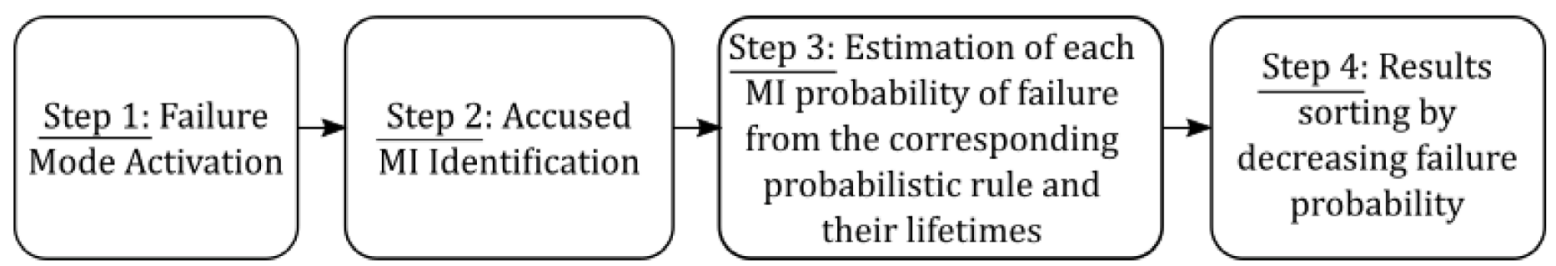

Fig. 4. The diagnosis process 4 steps.

order to define this probabilistic expert rule, a reliability analysis has been performed to model the failure rate of an equipment. However, this failure rate model depends on the type of equipment considered. Usually, the evolution of a physical equipment's reliability can be divided into three main periods. The first period is the running in period. This period represents the beginning of the equipment's lifetime where failures may occur due to production defects or installation issues. Next is the permanent period which corresponds to the later part of the lifetime. In this period, the failure rate is globally constant and low because the system is in its main phase of life. Random events may occur but independently of the lifetime and with low probability. The third period is the ageing period. During this period, the equipment's lifetime reaches its end. As a consequence, the risk of failure increases due to natural wearing. However, the three phases are not distinct in all types of equipment.

For instance the reliability of a mechanical equipment is different from an electronic equipment (Beleulmi et al., 2014). Mechanical systems usually have a consequent failure rate in the running in period and an increasing failure rate in the ageing period with no clear permanent period. Otherwise, in electronic systems the three periods are usually clearly distinct.

\subsection{Weibull distribution}

In the aeronautic field, reliability is a major concern. As a consequence, aircraft systems have been designed to be extremely reliable which led to relatively low failure occurrences. Several probabilistic distributions are often used in the literature like the Exponential distribution, the Log-Normal distribution or the Weibull distribution (Couallier et al., 2016). According to Beleulmi et al. (2014), the Weibull distribution is the universal distribution. Moreover, in the aeronautic field the three periods can be represented depending on the system. As such, the Weibull distribution has been chosen to model the equipment's reliability.

To model a bathtub-shaped failure rate with all three periods, a combination of two Weibull distributions is used Lai (2014) and Xie and Lai (1996). The density distribution $f$ and cumulative distribution $F$ functions are respectively given by:

$f(x)=\left(\left(\frac{k_{1}}{\lambda_{1}}\right)\left(\frac{x}{\lambda_{1}}\right)^{\left(k_{1}-1\right)}+\left(\frac{k_{2}}{\lambda_{2}}\right)\left(\frac{x}{\lambda_{2}}\right)^{\left(k_{2}-1\right)}\right)\left(e^{-\left(\frac{x}{\lambda_{1}}\right)^{k_{1}}} e^{-\left(\frac{x}{\lambda_{2}}\right)^{k_{2}}}\right)$

$F(x)=1-e^{-\left(\frac{x}{\lambda_{1}}\right)^{k_{1}}} e^{-\left(\frac{x}{\lambda_{2}}\right)^{k_{2}}}$

with $k_{1}>0, \lambda_{1}>0, k_{2}>0, \lambda_{2}>0$ respectively the shape and scale parameters of Weibull distributions.

To estimate the parameters of a Weibull distribution a regression method is performed based on Ordinary Least Square Estimator on the cumulative curve of empirical failures according to Zhang et al. (2008). However, in this case, no weights are attributed to failures. The corrected Akaike Information Criterion (AICc) is used to identify the best distribution between a traditional Weibull distribution and the one presented in (13). The choice of the AICc is motivated by the small size of the failures sample and its efficiency with regression models (Hurvich \& Tsai, 1989).
The AICc formula is given by:

$A I C c=2 p-\ln (L)+\frac{2 p(p+1)}{n-p-1}$

where $\mathrm{p}$ is the number of parameters ( $p=2$ for a simple Weibull distribution and $p=4$ for a combination of Weibull distributions), $n$ is the sample size and $L$ is the maximum value of likelihood function. The model with the smallest AICc is chosen. Finally, a KolmogorovSmirnov test is performed in accordance with Nwobi and Ugomma (2014) to check if the model with the estimated parameters follows a Weibull distribution. If the test of Kolmogorov-Smirnov rejects the Weibull distribution, an other distribution should be used to model the empirical curves. In this case study, the test has never rejected the Weibull distribution hypothesis.

\section{Experiments on the diagnosis process}

\subsection{Experiment's protocol}

In the previous sections, the concepts and structure of the HIN based ES have been defined. The aeronautical case study has been presented with the failure detection and diagnosis processes has applications. The diagnosis process relies on 4 steps has described in Fig. 4. The first step is the activation of a failure mode. This activation triggers the collect of accused MIs. These MIs should then be sorted by decreasing probability of failure after these probabilities have been computed. In this case study, the probabilistic rules used to estimate these probabilities are based on Weibull models.

In order to assess the presented ES diagnosis performance, the HIN, base of rules and control structure have been implemented using a tool called Digibrain (Activus) according to Section 3.

The documentation, maintenance and monitoring data have been structured according to the metamodel presented in Fig. 3. Entities are translated as nodes and relations as edges. An associate operator has been used to create and fill the nodes and edges from these different sources. A check operator can also control imported data quality.

The diagnosis process is performed using a projection operator between the failure modes and MIs. A failure mode with an activated status triggers a Read rule to collect the related MIs. An Update is then performed on each MI to calculate the probability of failure from the Weibull distributions and the MI's model and lifetime. Next, an update on the failure mode diagnosis attribute generates the list of affected MIs sorted by decreasing probability of failure. The projection operator can also be used to update an MI's attribute. Indeed, MI's lifetime and/or model should be updated after a repair, a replacement and after each use.

The following steps of this experiment consist in designing the Weibull models for each MI and run the diagnosis process before analyzing the results.

\subsection{Weibull models design}

The first objective of the diagnosis is to identify the model of the failed equipment from past maintenance records. The second objective is to identify the physical position of this failed equipment. The probabilistic rules allowing to identify the failed equipment model and 


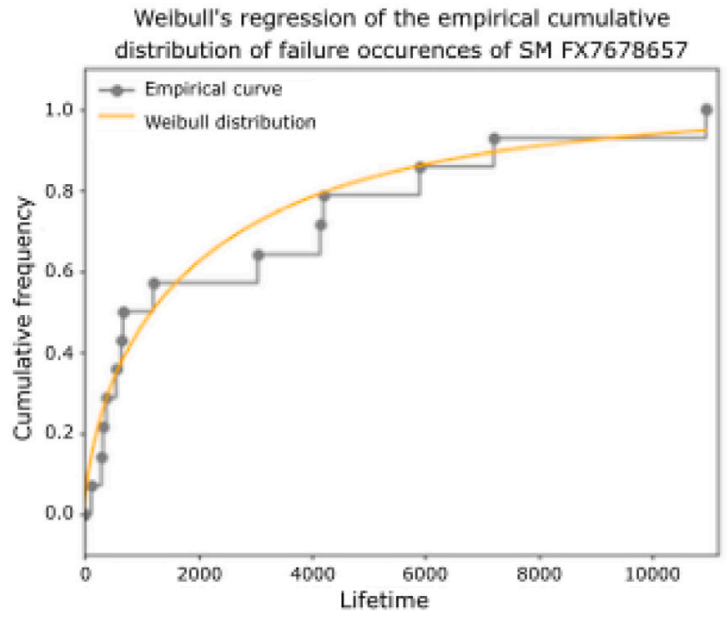

Weibull's regression of the empirical cumulative distribution of failure occurences of SM 12755553-55



Weibull's regression of the empirical cumulative



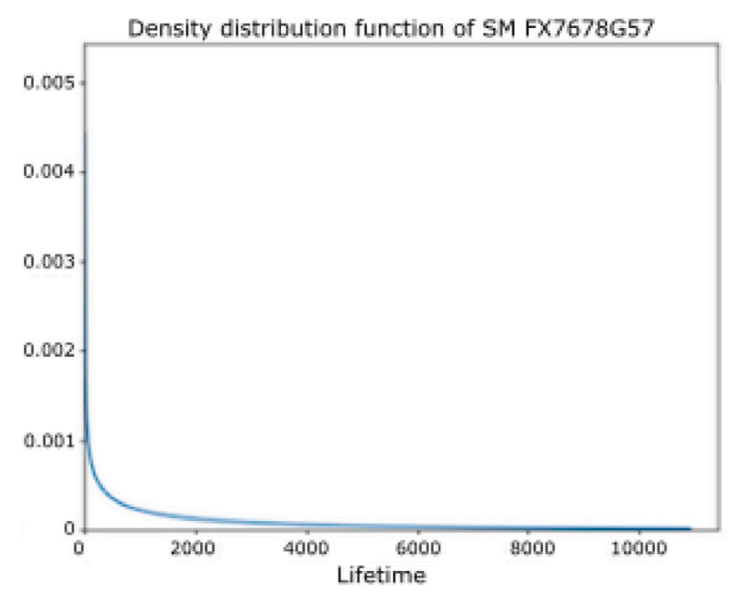
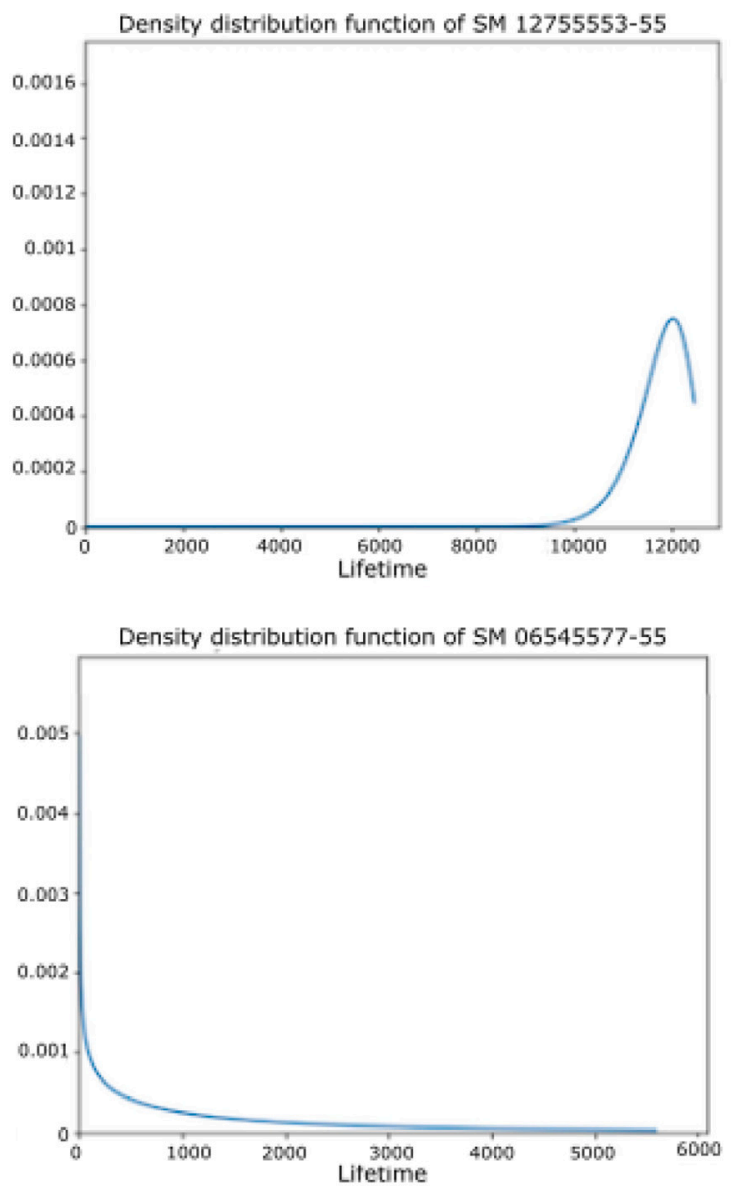

Fig. 5. Failures' modeling on Spare Model by Weibull distributions.

position have been designed from an aeronautic maintenance data set containing over 70 planes of the same type over 5 years (from several airlines).

In this perspective, two empirical approaches have been designed. The first approach, named Spare Model (SM), models the failure occurrences by spare model over the equipment's lifetime without considering their physical position in the aircraft. The second approach, named Position Spare Model (PSM), takes this feature into account by modeling the failure occurrences by MI and Spare Model. Indeed, several equipments with the same SM can be located at different positions in the same time on the aircraft. However, a MI refers to an equipment located in a specific position.

Hence, considering an equal amount of failures, the second approach designs more models with less empirical points by model. However, this approach is more detailed as it identifies the failed component on an individual, physical, level. In each case, we chose to model failure instants with a Weibull distribution when there were at least three failure occurrences. Otherwise, the failure rate is constant and close to zero. The results of the modeling are presented in Fig. 5. These figures illustrate the three different behaviors (running in period, ageing period or permanent failure period) for each approach. 


\subsection{Evaluation protocol}

In order to evaluate the performance of the graph with these models, records of failure modes have been gathered over a year with the related failed equipment identified.

The aim of the diagnostic is to provide a list of potentially failed Equipment Models ordered by decreasing failure's probability. This list is compared to the real breakdowns to evaluate the diagnostics's accuracy. The experiment is composed of 5 steps. The first step is to identify the MI from the recorded failure mode. The second step is to identify the corresponding degradation model for each MI. The third step is to calculate the maintainable item's failure's probability from the degradation model and the MI's lifetime at the failure occurrence date. The fourth step is to generate the list of potentially failed equipments by decreasing failure probability. Finally, the diagnostics performance is evaluated by observing the ranking of the failed equipment in the previous list.

Noticed that the diagnostic based on the Spare Model can provide the same breakdown probability to different MI if their models and lifetimes are identical. This means that similar MIs installed at the same time on the aircraft will be given the same ranking in the diagnostic result list. However, when using the PSM where the physical position is considered, two MIs, even similar, do not refer to the same curve. As such their breakdown probability and rankings differ.

Figs. 6 and 7 illustrate the ranking of the real failed equipment in the list returned by the diagnostic based on the Spare Model. Colors in the histograms represent the total number of equipment in the list returned by the diagnostic, that is to say the total number of MIs returned by the graph. For instance, the red part with the rank " 1 " corresponds to the number of failure modes where the failed equipment has been correctly identified among 2 possibilities. MIs with the ranking " 0 " represent the situation where a single MI possibly responsible for the failure according to the graph. In this case, which represents $36 \%$, the Weibull models are not needed.

\subsection{Evaluation results}

Fig. 6 illustrates results using Weibull model on Sparse Model. It allows to predict correctly $\mathbf{7 1 \%}$ in the first rank in the returned list. The diagnostic obtains an accuracy of $\mathbf{9 4 \%}$ by observing the two first propositions only thanks to the probabilistic rule.

In Fig. 7 , the ranks " 0 " and " 1 " have been aggregated to represent the actual performance of the diagnostic. The global accuracy of the diagnostic is $\mathbf{8 1 \%}$ looking the first MI returned in the list and $\mathbf{9 6 \%}$ looking the two first items with the greatest probability of breaking down.

The same approach has been respectively used to represent the results obtained with the PSM. As shown in Fig. 8, 36\% of the results are given directly by the graph. The PSM predicts correctly $\mathbf{5 9 \%}$ more ( $81 \%$ more in top 2) when a choice is needed and by aggregating all correct answers in Fig. 9, global accuracy is now of $\mathbf{7 4 \%}$ (88\% in top 2).

The Spare Model and PSM both have their advantages. While the Spare Model is less detailed as it points the model of the failed equipment and not the specific part, it offers better performance and can be sufficient from a logistic perspective to improve the supply chain management and maintenance scheduling for instance. At the same time, the PSM is more interesting for the maintenance operators as it indicates which equipment to test at a physical level, saving time in the repair process.

As a conclusion, using these probabilistic rules, the detection of the failed item improves from $36 \%$ with the physical and functional descriptions alone to $81 \%$ with the SM and $74 \%$ with the PSM. Furthermore, while some failed equipments are ranked in the second place or below in the diagnostics list, it may not mean that equipments ranked before have not failed. Further works could focus on considering this aspect in the results' evaluation.



Fig. 6. Evaluation of probabilistic rule using modeling on Spare Model on all airlines.



Fig. 7. Global results of the diagnostic using modeling on Spare Model on all airlines.

\section{Conclusion and perspectives}

The maintenance domain is confronted to increasing requirements in reliability and cost reduction driving the need for more efficient maintenance policies. In this context, condition-based maintenance, prognostics and health management are promising as they allow a better evaluation of the systems' degradation and environmental conditions. These strategies are mainly implemented based on historical data records using machine learning or based on capitalized expert knowledge through an expert system.

This paper proposes an expert system framework addressing modern ES limitations in efficiency, scalability and applicability. It is based on an heterogeneous information network in charge of supporting and manage the ES base of fact, base of rules and control structure. The choice of the HIN addresses the efficiency and scalability issues of modern ES which are not adapted to complex and dynamic systems. A generic meta-model for CBM is used to answer the adaptability requirements for these systems. A case study on an aircraft is presented 


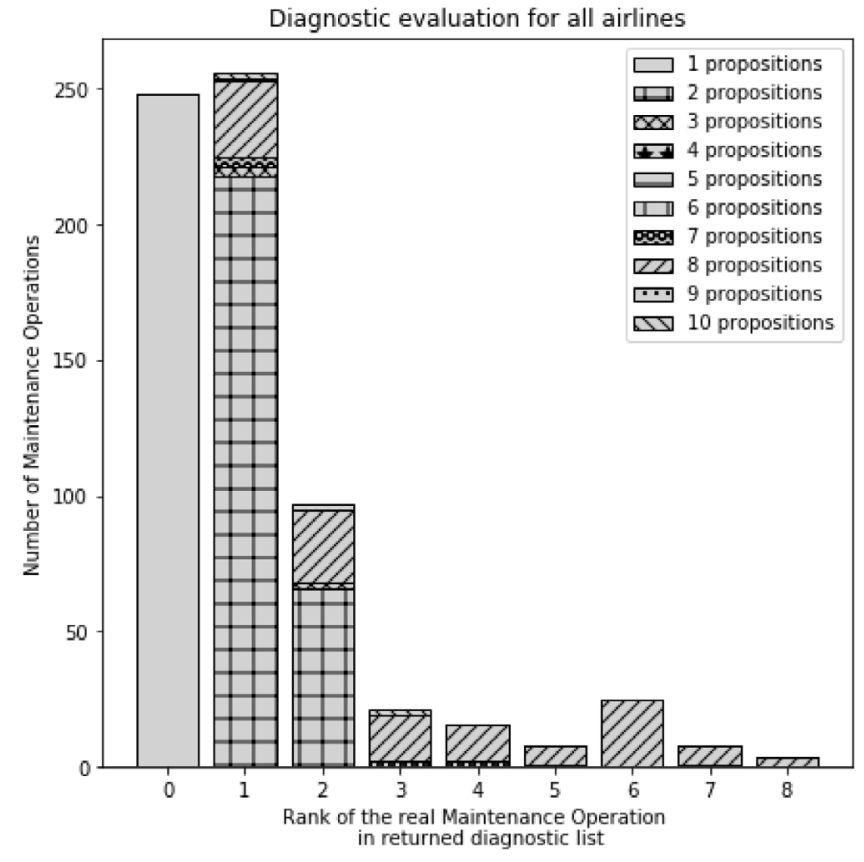

Fig. 8. Evaluation of probabilistic rule using modeling on Position and Spare Model on all airlines.

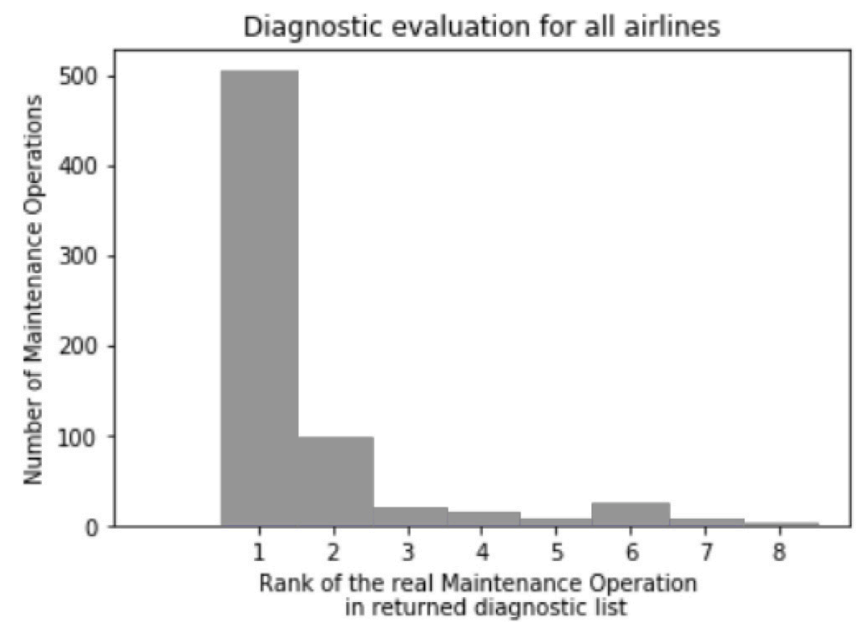

Fig. 9. Global results of the diagnostic using modeling on Position and Spare Model on all airlines.

to illustrate this frameworks' implementation and provide an example of probabilistic rule based on Weibull distribution for diagnostics.

Despite the frameworks' promising results, some limits and perspectives still need to be addressed. First, the availability of sensor and documentation resources are a necessity for the framework to be implemented. This limit is inherent to most condition based maintenance solutions and can be considered a critical limit to the research development in the field. A second limit is the exploitation and structuring of documentation resources into a base of facts. Some technical documentation being in paper or pdf format, processing these resources to generate an HIN can be considered as a challenge and a strong perspective. An other perspective should be to develop more case studies to challenge the frameworks adaptation ability to different systems. Indeed, as the metamodel was designed to be generic and adaptable, this genericity should be challenged by testing other systems with more nodes and relations to evaluate the overall performance. Finally, more complex models should be integrated to improve the diagnostics and prognostics performance. In particular, several works focused on developing diagnostics and prognostics models for gas turbine engine (Hanachi et al., 2018; Puggina \& Venturini, 2012; Roemer \& Kacprzynski, 2000; Zaidan et al., 2016). A case study could be designed to implement and test these models with C-MAPSS data.

\section{CRediT authorship contribution statement}

Alexandre Sarazin: Conception and design of study, Acquisition of data, Writing - original draft. Jérémy Bascans: Conception and design of study, Acquisition of data, Writing - original draft. JeanBaptiste Sciau: Conception and design of study, Writing - original draft. Jiefu Song: Conception and design of study, Writing - original draft. Bruno Supiot: Analysis and/or interpretation of data, Writing review \& editing. Aurélie Montarnal: Analysis and/or interpretation of data, Writing - review \& editing. Xavier Lorca: Analysis and/or interpretation of data, Writing - review \& editing. Sébastien Truptil: Analysis and/or interpretation of data, Writing - review \& editing.

\section{Declaration of competing interest}

The authors declare that they have no known competing financial interests or personal relationships that could have appeared to influence the work reported in this paper.

\section{Acknowledgments}

All authors approved the version of the manuscript to be published.

\section{References}

Abu-Nasser, B. (2017). Medical expert systems survey: Ssrn scholarly paper id 3082734, Rochester, NY: Social Science Research Network, URL: https://papers.ssrn.com/ abstract $=3082734$.

Activus (0000). Activus Software. URL: https://www.activus-group.fr/presentation/ activus-software.html.

AFNOR (2018). Nf en 13306 - maintenance - terminologie de la maintenance.

Agrawal, R., Bayardo, R., \& Srikant, R. (2000). Athena: Mining-based interactive management of text databases. In International conference on extending database technology (pp. 365-379). Springer.

Angelova, R., Kasneci, G., \& Weikum, G. (2012). Graffiti: graph-based classification in heterogeneous networks. World Wide Web, 15(2), 139-170, Publisher: Springer.

Bayoumi, A., Goodman, N., Shah, R., Eisner, L., Grant, L., Keller, J., \& Center, C.B. M. (2008). Conditioned-based maintenance at USc-part IV: Examination and cost-benefit analysis of the CBM process. In AHS international specialists' meeting on condition based maintenance, huntsville, al.

Beleulmi, S., Bellaouar, A., \& Benidir, M. (2014). Reliability modeling of transport systems: Influence of the running-in period of life. Physics Procedia, 55, 75-82, Publisher: Elsevier.

Chein, M., Mugnier, M.-L., \& Simonet, G. (1998). Nested graphs: A graph-based knowledge representation model with FOL semantics. In KR (pp. 524-535).

Cheng, S., Azarian, M. H., \& Pecht, M. G. (2010). Sensor systems for prognostics and health management. Sensors, 10(6), 5774-5797. http://dx.doi.org/10. 3390/s100605774, URL: https://www.mdpi.com/1424-8220/10/6/5774 Number: 6 Publisher: Molecular Diversity Preservation International.

Corset, F., Celeux, G., \& Lannoy, A. (2003). Introduction du retour d'expérience dans les réseaux bayésiens. In Qualita. Nancy, France: URL: https://hal.archivesouvertes.fr/hal-02298326.

Couallier, V., Denis, L., \& Bayle, F. (2016). MTBF computations for aeronautical maintained repairable systems with dormant failure mode of control device. In 20 e congrès de maîtrise des risques et de sureté de fonctionnement, communication $4 e$ $/ 2$ (pp. 1-8).

Davis, D., Lichtenwalter, R., \& Chawla, N. V. (2011). Multi-relational link prediction in heterogeneous information networks. In 2011 international conference on advances in social networks analysis and mining (pp. 281-288). IEEE.

Guillén, A. J., Crespo, A., Gómez, J. F., \& Sanz, M. D. (2016). A framework for effective management of condition based maintenance programs in the context of industrial development of E-maintenance strategies. Computers in Industry, 82, 170-185. http://dx.doi.org/10.1016/j.compind.2016.07.003, URL: http://www.sciencedirect. com/science/article/pii/S0166361516301178.

Gündüz, D., de Kerret, P., Sidiropoulos, N. D., Gesbert, D., Murthy, C. R., \& van der Schaar, M. (2019). Machine learning in the air. IEEE Journal on Selected Areas in Communications, 37(10), 2184-2199. 
Hanachi, H., Mechefske, C., Liu, J., Banerjee, A., \& Chen, Y. (2018). Performancebased gas turbine health monitoring, diagnostics, and prognostics: A survey. IEEE Transactions on Reliability, 67(3), 1340-1363.

Hurvich, C. M., \& Tsai, C.-L. (1989). Regression and time series model selection in small samples. Biometrika, 76(2), 297-307.

Huynh, K. T. (2020). Modeling past-dependent partial repairs for condition-based maintenance of continuously deteriorating systems. European Journal of Operational Research, 280(1), 152-163.

Jackson, P. (1998). Introduction to expert systems (3rd ed.). Boston, MA, USA: Addison-Wesley Longman Publishing Co., Inc..

Jouin, M., Gouriveau, R., Hissel, D., Péra, M.-C., \& Zerhouni, N. (2013). Prognostics and health management of PEMFC - state of the art and remaining challenges. International Journal of Hydrocarbon Engineering, 38(35), 15307-15317. http://dx.doi. org/10.1016/j.ijhydene.2013.09.051, URL: http://www.sciencedirect.com/science/ article/pii/S036031991302274X.

Lai, C.-D. (2014). Generalized weibull distributions. In Generalized weibull distributions (pp. 107-111). Springer.

Lee, J., Wu, F., Zhao, W., Ghaffari, M., Liao, L., \& Siegel, D. (2014). Prognostics and health management design for rotary machinery systems-Reviews, methodology and applications. Mechanical Systems and Signal Processing, 42(1), 314-334. http: //dx.doi.org/10.1016/j.ymssp.2013.06.004, URL: http://www.sciencedirect.com/ science/article/pii/S0888327013002860.

Levine, P., \& Pomerol, J.-C. (1990). Systèmes interactifs d'aide à la décision et systèmes experts. Hermès, URL: https://infoscience.epfl.ch/record/2917.

Liebowitz, J. (1995). Expert systems: A short introduction. Engineering Fracture Mechanics, 50(5-6), 601-607.

Numanoğlu, M. F., \& Ekmekçi, İ. (2020). Analysis of the periodic maintenance costs of aircraft fleet. Journal of International Trade, Logistics and Law, 6(1), 126.

Nwobi, F. N., \& Ugomma, C. A. (2014). A comparison of methods for the estimation of Weibull distribution parameters. Metodoloski Zvezki, 11(1), 65, Publisher: Anuska Ferligoj.

Puggina, N., \& Venturini, M. (2012). Development of a statistical methodology for gas turbine prognostics. Journal of Engineering for Gas Turbines and Power, 134(2).

Rajabi, M., Hossani, S., \& Dehghani, F. (2019). A literature review on current approaches and applications of fuzzy expert systems. URL: http://arxiv.org/abs/ 1909.08794 arXiv:1909.08794 [Cs] arXiv:1909.08794.
Roemer, M. J., \& Kacprzynski, G. J. (2000). Advanced diagnostics and prognostics for gas turbine engine risk assessment. In 2000 ieee aerospace conference. proceedings (cat. no. 00th8484), Vol. 6 (pp. 345-353). IEEE.

Roussey, C. (2001). Un modèle de graphe pour la recherche d'information multilingue. In INFORSID (pp. 419-441).

Salameh, J. P., Cauet, S., Etien, E., Sakout, A., \& Rambault, L. (2018). Gearbox condition monitoring in wind turbines: A review. Mechanical Systems and Signal Processing, 111, 251-264.

Sheng, V. S., \& Zhang, J. (2019). Machine learning with crowdsourcing: A brief summary of the past research and future directions. In Proceedings of the aaa conference on artificial intelligence, Vol. 33 (pp. 9837-9843).

Sowa, J. (1983). Conceptual structures: information processing in mind and machine. Reading, MA, United States: Addison-Wesley Pub., URL: https://www.osti.gov/ servlets/purl/5673179.

Sun, Y., \& Han, J. (2012). Mining heterogeneous information networks: principles and methodologies. Synthesis Lectures on Data Mining and Knowledge Discovery, 3(2), $1-159$.

Xiaoxue, L., Xuesong, B., Longhe, W., Bingyuan, R., Shuhan, L., \& Lin, L. (2019). Review and trend analysis of knowledge graphs for crop pest and diseases. IEEE Access, 7, 62251-62264. http://dx.doi.org/10.1109/ACCESS.2019.2915987, Conference Name: IEEE Access.

Xie, M., \& Lai, C. D. (1996). Reliability analysis using an additive Weibull model with bathtub-shaped failure rate function. Reliability Engineering \& System Safety, 52(1), 87-93.

Yanase, J., \& Triantaphyllou, E. (2019). A systematic survey of computer-aided diagnosis in medicine: Past and present developments. Expert Systems with Applications, 138, Article 112821. http://dx.doi.org/10.1016/j.eswa.2019.112821, URL: http: //www.sciencedirect.com/science/article/pii/S0957417419305238.

Zaidan, M. A., Harrison, R. F., Mills, A. R., \& Fleming, P. J. (2015). BayesIan hierarchical models for aerospace gas turbine engine prognostics. Expert Systems with Applications, 42(1), 539-553.

Zaidan, M. A., Mills, A. R., Harrison, R. F., \& Fleming, P. J. (2016). Gas turbine engine prognostics using Bayesian hierarchical models: A variational approach. Mechanical Systems and Signal Processing, 70, 120-140.

Zhang, L., Xie, M., \& Tang, L. (2008). On weighted least squares estimation for the parameters of Weibull distribution. In Recent advances in reliability and quality in design (pp. 57-84). Springer. 